\title{
DISCIPLINA DE PROJETO DE SOFTWARE DO CURSO DE SISTEMAS DE INFORMAÇÃO: relato de experiências realizadas
}

Cristiano BERTOLINI ${ }^{1}$

Sidnei Renato SILVEIRA ${ }^{2}$

\begin{abstract}
${ }^{1}$ Departamento de Tecnologia da Informação - UFSM - Universidade Federal de Santa Maria - Campus Frederico Westphalen - RS. cristiano.bertolini@ufsm.br

${ }^{2}$ Departamento de Tecnologia da Informação - UFSM - Universidade Federal de Santa Maria - Campus Frederico Westphalen - RS. sidneirenato.silveira@gmail.com
\end{abstract}

Recebido em: 12/04/2016 - Aprovado em: 12/10/2017 - Disponibilizado em: 30/12/2017

\begin{abstract}
RESUMO:
Este artigo apresenta o relato de uma experiência realizada na disciplina de Projeto de Software, do curso de Bacharelado em Sistemas de Informação da <instituição>. A disciplina de Projeto de Software possui o objetivo de estimular o desenvolvimento de sistemas computacionais, tendo como base os conceitos já estudados nas disciplinas dos primeiros 2 anos do curso. Neste artigo, são apresentadas as experiências realizadas em 3 edições da disciplina, onde cada edição contou com uma metodologia própria. Como principais resultados obtidos têm-se o desenvolvimento de sistemas de informação completos, artigos publicados e sistemas desenvolvidos para clientes reais.

Palavras-Chave: Projeto de Software, Sistemas de Informação, Desenvolvimento de Software, Educação em Informática
\end{abstract}

\section{ABSTRACT:}

This paper presents an experience in Software Development Project course at Information Systems Undergraduate Program from <institution>. The main goal of the Software Development Project course is to stimulate the software development based on the students' Knowledge from the 3 initial years of the Information Systems Undergraduate Program. In this paper, it is reported the experience of the 3 course editions, where each edition has its own methodology. As the main results, there are deployed complete information systems, published papers and systems developed for real clients.

Keywords: Software Project, Information Systems, Software Development, Computer Education

\section{INTRODUÇÃO}

O curso de Bacharelado em Sistemas de Informação (SI) da <instituição> oferece uma formação voltada para a pesquisa e para o desenvolvimento na área de Sistemas de Informação, visando o projeto e construção de software e serviços computacionais, além da aplicação de sistemas computacionais em outras áreas(MEC, 2012; ref05, 2009).

Uma das disciplinas do curso denomina-se Projeto de Software e possui como objetivo: (i) aplicar os conceitos e técnicas de Projeto e Gerência de Software, dando ênfase à construção piloto de aplicações, produtos ou serviços computacionais (PRESSMAN, 2011; PADUA, 2009; SOMMERVILLE, 2007); (ii) usar técnicas de trabalho em grupo; (iii) e identificar um problema real a ser resolvido. A disciplina possui uma carga horária de 8 horas semanais, totalizando 120 horas no semestre com dois encontros por semana.

Uma das propostas aplicadas envolve o desenvolvimento da disciplina com um enfoque diferente. Na primeira edição foram abordadas metodologias mais tradicionais de 
desenvolvimento de software como o RUP (RationalUnifiedProcess) e vários artefatos foram gerados; na segunda edição da disciplina foram abordadas bases de dados abertas e metodologias ágeis e, na terceira edição, foram desenvolvidos projetos para clientes reais de diferentes setores da universidade e outros departamentos. Explorar diferentes experiências na disciplina é um desafio e requer, a cada edição, uma mudança completa no cronograma, atividades e formas de realizar a avaliação do rendimento acadêmico.

Neste contexto, este artigo apresenta as diferentes abordagens e resultados de cada uma das 3 edições da disciplina de Projeto de Software no curso de Sistemas de informação. Para cada uma das edições da disciplina uma metodologia diferente foi abordada. $\mathrm{O}$ artigo está organizado da seguinte forma: a Seção 2 apresenta os trabalhos relacionados; a Seção 3 apresenta a metodologia e histórico de cada uma das edições da disciplina de Projeto de Software; a Seção 4 apresenta os resultados obtidos e as principais lições aprendidas. Por fim, são apresentadas as principais conclusões e referências bibliográficas.

\section{TRABALHOS RELACIONADOS}

Com relação aos trabalhos envolvendo a Educação Superior em Informática, a SBC (Sociedade Brasileira de Computação) promove, anualmente, o WEI (Workshop sobre Educação em Computação). Este evento tem, por objetivo, debater diferentes temas relacionados ao ensino de Computação e Informática, constituindo-se em um importante fórum para apresentação de trabalhos científicos, reuniões e debates entre pesquisadores, docentes, discentes, coordenadores de cursos e demais interessados na melhoria da Educação em Computação (SBC, 2014). Além do WEI, no Rio Grande do Sul, um grupo de docentes organizou o WEI Tchê. Este evento foi organizado juntamente ao SEMINFO (Seminário de Informática) (http://www.seminfo.com.br), com o apoio da SBC.

Um grande número de trabalhos apresentados nas diferentes edições do WEI envolve a construção de ferramentas para simulação e/ou para o auxílio no aprendizado de programação, além de trabalhos que envolvem a discussão sobre estruturas e/ou matrizes curriculares. Outra área que tem obtido destaque é a que envolve a aplicação de jogos nos processos de ensino e de aprendizagem.

Destacando-se os trabalhos relacionados a este artigo, apresentados no WEI, na edição de 2015 foi apresentado um relato de experiência envolvendo a aplicação demétodos ágeis no desenvolvimento de software. Diferentemente do trabalho aqui apresentado, que envolve uma disciplina, este relato envolve a aplicação de métodos ágeis em um Núcleo de Práticas Acadêmico, em 
que os alunos são estagiários (ALMENDRA;

MAGALHÃES; ALMEIDA, 2015).

Barth, Burd e Pimentel (2012) desenvolveram um currículo de um Curso Superior de Tecnologia contendo disciplinas relacionadas à participação dos alunos em um Escritório de Projetos. Este escritório simula um ambiente real de projetos de software, sendo uma disciplina obrigatória para todos os alunos. O nível de complexidade dos projetos depende do semestre em que o aluno está matriculado. O escritório de projetos funciona de forma similar a uma Empresa Júnior dos Cursos de Administração.

A proposta de "residência em desenvolvimento de software" é apresentada por Borges, Carvalho e Moraes (2012). A residência em desenvolvimento de software faz parte de um programa de extensão que integra os três pilares da Educação Superior: ensino, pesquisa e extensão. No caso da residência em desenvolvimento de software, o aluno vivencia a experiência profissional em um ambiente similar ao mercado de trabalho, atendendo às necessidades dos clientes de uma "fábrica de software acadêmica". Diferentemente do trabalho aqui apresentado, estas atividades não são desenvolvidas de forma curricular, ou seja, ligadas a uma determinada disciplina.

\section{METODOLOGIA E HISTÓRICO DA DISCIPLINA DE PROJETO DE SOFTWARE}

A disciplina de Projeto de Software do Curso de SI da <Instituição> contou com 3 edições, realizadas nos anos de 2013, 2014 e 2015. A Tabela 1 apresenta uma estatística de alunos matriculados nas 3 (três) edições da disciplina. Observa-se que a quantidade de alunos vem crescendo em cada uma das edições e apenas houve reprovações por frequência nas duas primeiras edições.

Tabela 1: Relação das edições da disciplina e alunos matriculados

\begin{tabular}{|c|c|c|c|}
\hline Edição & Ano & $\begin{array}{c}\text { Quantidade } \\
\text { de Alunos }\end{array}$ & Reprovados \\
\hline 1 & 2013 & 11 & 1 (por frequência) \\
\hline 2 & 2014 & 17 & 1 (por frequência) \\
\hline 3 & 2015 & 32 & - \\
\hline
\end{tabular}

Semestralmente, a Coordenação do Curso aplica um instrumento visando coletar as impressões dos alunos com relação ao processo acadêmico, dentro do contexto da avaliação institucional. Este instrumento visa identificar a opinião dos alunos sobre $\mathrm{o}$ trabalho dos professores em cada uma das disciplinas. A Tabela 2 apresenta os resultados da avaliação do processo acadêmico da disciplina de Projeto de Software. As respostas das 3 edições foram agrupadas, totalizando 36 alunos respondentes que representam $60 \%$ dos alunos matriculados nestas edições.

Analisando os resultados da Tabela 2, 
verifica-se que a avaliação da disciplina é positiva, considerando-se os percentuais de respostas muito bom e bom para os critérios avaliados.

A seguir são apresentados os detalhes de cada uma das edições da disciplina. A característica comum da disciplina de Projeto de Software é o desenvolvimento de um projeto em grupo onde os alunos devem utilizar os conhecimentos adquiridos no decorrer do curso e colocar em prática as técnicas e conceitos de Engenharia de Software e Programação, entre outras áreas.

Tabela 2: Resultado da avaliação da disciplina de Projeto de Software.

\begin{tabular}{|c|c|c|c|c|c|c|c|c|c|c|c|c|}
\hline & \multicolumn{2}{|c|}{$\begin{array}{l}\text { Muito } \\
\text { Bom }\end{array}$} & \multicolumn{2}{|c|}{ Bom } & \multicolumn{2}{|c|}{ Indiferente } & \multicolumn{2}{|c|}{ Regular } & \multicolumn{2}{|c|}{$\begin{array}{l}\text { Não } \\
\text { Satisfatório }\end{array}$} & \multicolumn{2}{|c|}{$\begin{array}{c}\text { Sem } \\
\text { condições } \\
\text { de opinar }\end{array}$} \\
\hline & $\mathrm{f}$ & $\%$ & f & $\%$ & $\mathrm{f}$ & $\%$ & $\mathrm{f}$ & $\%$ & $\mathrm{f}$ & $\%$ & $\mathrm{f}$ & $\%$ \\
\hline $\begin{array}{l}\text { Domínio do conteúdo por } \\
\text { parte do professor }\end{array}$ & 20 & 55,56 & 14 & 38,89 & 2 & 5,56 & - & - & - & - & - & - \\
\hline $\begin{array}{l}\text { Incentivo a participação dos } \\
\text { alunos em aula }\end{array}$ & 13 & 36,11 & 20 & 55,56 & 1 & 2,78 & 2 & 5,56 & - & - & - & - \\
\hline $\begin{array}{l}\text { Relacionamento com os } \\
\text { alunos }\end{array}$ & 21 & 58,33 & 10 & 27,78 & 1 & 2,78 & 2 & 5,56 & 2 & 5,56 & - & - \\
\hline $\begin{array}{c}\text { Motivação e interesse } \\
\text { demonstrado pelo professor } \\
\text { durante em sala de aula }\end{array}$ & 18 & 50,00 & 15 & 41,67 & 2 & 5,56 & 1 & 2,78 & - & - & - & - \\
\hline $\begin{array}{l}\text { Praticas pedagógicas } \\
\text { empregadas }\end{array}$ & 12 & 33,33 & 17 & 47,22 & 2 & 5,56 & 3 & 8,33 & 2 & 5,56 & - & - \\
\hline $\begin{array}{l}\text { Instrumentos e critérios de } \\
\text { avaliação utilizados }\end{array}$ & 13 & 36,11 & 18 & 50,00 & 3 & 8,33 & 1 & 2,78 & 1 & 2,78 & - & - \\
\hline $\begin{array}{c}\text { Dialogo entre professor e } \\
\text { alunos }\end{array}$ & 20 & 55,56 & 11 & 30,56 & 1 & 2,78 & 3 & 8,33 & 1 & 2,78 & - & - \\
\hline
\end{tabular}

Edição da Disciplina de Projeto de Software no Ano de 2013

A edição da disciplina de Projeto de Software no segundo semestre de 2013 contou com 11 alunos matriculados. Os alunos foram divididos em 3 grupos, sendo dois grupos de 4 alunos e um de 3 alunos.

Esta edição tinha por objetivo possibilitar que os alunos desenvolvessem um Sistema de Informação a sua escolha seguindo o processo de desenvolvimento
RUP (RationalUnifiedProcess). Com o tema livre, cada grupo pôde escolher qual sistema seria desenvolvido, bem como em qual tecnologia (linguagem de programação, framework, banco de dados, etc.). No entanto, os seguintes requisitos mínimos deveriam ser seguidos:

- Todos os artefatos gerados deveriam ser disponibilizados no sourceforge;

- Cada grupo deveria adicionar o professor no seu repositório no 
sourceforge, para que o mesmo realizasse o acompanhamento;

- Semanalmente o professor verificava a evolução dos projetos, e o mesmo acompanhava a evolução via sourceforge. As dúvidas, sugestões, problemas e inconsistências eram reportados durante as aulas presenciais.

- Para cada artefato gerado foi proposto um template (modelo) que deveria ser seguido pelos grupos;

A disciplina contou com aulas expositivas e aulas para o desenvolvimento dos projetos. A ideia era rever conceitos básicos de Engenharia de Software.

Com base nas aulas de revisão e novos conceitos apresentados aos alunos sobre Engenharia de Software, cada grupo ficou responsável em elaborador os seguintes artefatos: Plano de Negócios; Plano de Projetos; Especificação de requisitos no formato de Casos de uso; Documento de Casos de Testes; documentação do código; Matriz de Rastreabilidade; Manual do Usuário e Release Notes contendo todos os requisitos implementados e defeitos não corrigidos. $\mathrm{O}$ resultado final de cada projeto foi um sistema funcional com todos esses artefatos atualizados e consistentes.

Os seguintes projetos foram desenvolvidos:

- Sistema de Atenção Básica Saúde e Social: sistema que possibilita cadastrar agentes de saúde e social para que os mesmos possam realizar as entrevistas e coleta de dados via tablet, onde no final do dia, eles alimentem o banco de dados do sistema com as informações dos seus atendimentos, para possíveis consultas posteriormente. $\mathrm{O}$ projeto pode ser encontrado em <link do projeto omitido para a avaliação cega>.

- Meus Anúncios: sistema para a criação de anúncios para uma microrregião no interior do Brasil que viesse suprir as necessidades de anúncios online na região, como venda e compra de móveis, carros, etc. $\mathrm{O}$ projeto pode ser encontrado em $<$ link do projeto omitido para a avaliação cega>.

\section{- Sistema de Gerenciamento}

Acadêmico: sistema que visa atender secretarias, professores, comunidade e alunos de uma instituição de ensino, utilizando ferramentas de gerência de requisições, controle de usuários, registros de aulas e notas, noticia e reserva de salas e laboratórios. O projeto pode ser encontrado em <link do projeto omitido para a avaliação cega>.

Edição da Disciplina de Projeto de Software no Ano de 2014

A edição de 2014 da disciplina contou 
com 17 alunos matriculados. Os alunos foram divididos em 2 grupos de 6 e um grupo de 5 integrantes cada.Nesta edição da disciplina o objetivo era desenvolver um sistema funcional que utilizasse bases de dados da área da saúde (http://dados.gov.br/). Os critérios para o desenvolvimento do sistema foram definidos pelo professor e os grupos de alunos escolheram apenas um dos temas sugeridos (Bases de Dados Abertas do Governo Federal) para desenvolver o seu sistema. Os seguintes requisitos mínimos deveriam ser seguidos:

- Utilizar as tecnologias: Django (Python) e banco de dados Postgres ou MySQL;

- Empregar pelo menos uma base de dados aberta;

- Utilizar a API (ApplicationProgram Interface) do GoogleMaps para a visualização dos dados;

- Descrever os requisitos por meio de especificação de casos de uso e diagramas

UML

\section{(UnifiedModelingLanguage);}

- O sistema deveria estar funcional na entrega final.

A disciplina contou com aulas expositivas iniciais sobre bases de dados abertas e o framework Django, e aulas para o desenvolvimento dos projetos. A ideia era focar nas tecnologias e nas bases de dados abertas escolhidas pelos grupos. Os seguintes projetos foram desenvolvidos:
- Incidência de Acidentes de Trabalho em segurados da previdência social: 0 principal objetivo deste projeto foi implementar um aplicativo que facilitasse a visualização dos dados relacionados a acidentes de trabalho. $\mathrm{O}$ projeto também foi publicado em um evento por meio de um artigo científico $<$ ref01>.

- Incidência de Malária no Brasil: o principal objetivo desse projeto foi implementar um sistema para a visualização e controle dos dados da malária de forma dinâmica por meio de filtros. O projeto também foi publicado em um evento por meio de um artigo científico <ref02〉.

- Sistema de Consulta à Incidência de

Doenças: 0 sistema foi desenvolvimento de uma forma genérica, onde todas as doenças que possuem dados abertos e fornecidos pelo governo possam ser inseridas, facilitando as avaliações e desenvolvimento de políticas, planos e programas de saúde.

Durante o semestre houve a avaliação continuada por meio de reuniões semanais do tipo SCRUM. Nas reuniões cada aluno respondia a 3 perguntas: (i) que fez na semana anterior; (ii) o que fará até a próxima semana; e (iii) se teve algum problema que o impediu de realizar alguma de suas atividades. 
Edição da Disciplina de Projeto de Software no Ano de 2015

Esta edição contou com 32 alunos matriculados. Os alunos foram divididos em 6 grupos com 5 ou 6 alunos em cada. O objetivo era desenvolver um sistema funcional para algum setor da universidade ou grupo de pesquisa de outro departamento. Antes da disciplina iniciar, o professor convidou várias pessoas da universidade (denominados clientes), que tinham alguma demanda no desenvolvimento de software. Selecionados os projetos de cada cliente, os mesmos foram atribuídos aos grupos. Os seguintes requisitos mínimos deveriam ser seguidos:

- Os sistemas desenvolvidos deveriam ser voltados à plataforma $w e b$;

- Todo o desenvolvimento deveria ser feito usando as ferramentas Github (https://github.com/) e Trello (https://trello.com/);

- Forma definidas 4 entregas, sendo que em todas elas deveriam ser entregues requisitos funcionais implementados, testados e com documentação atualizada.

Nesta edição, o formato da disciplina foi um pouco diferente. Metade da carga horaria da disciplina foi realizada na modalidade a distância, ou seja, os encontros presenciais ocorriam uma vez por semana e o restante era realizado a distância pelos alunos.
Uma das razões para a aplicação de recursos de $\mathrm{EaD}$ (Educação a Distância), foi a utilização de plataformas para desenvolvimento distribuído de software como o Github e o Trello. Os projetos propostos pelos clientes foram:

- Sistema de Gerenciamento de Patrimônio: atualmente, o sistema da <universidade> gera relatórios com a lista de patrimônio de cada departamento. No entanto, é preciso fazer um controle do local (em cada campus) onde o patrimônio encontrase (Bloco e Sala). O Sistema deveria importar os dados em formato CSV (Comma-SeparatedValues) ou em planilhas do Excel e possibilitar o gerenciamento do local para cada um dos itens do patrimônio;

- Sistema de Visualização de Análise de Água: o sistema deveria importar os dados da análise de água de diferentes locais mostrando, entre outras características, em quais locais a água está própria para consumo humano. Deveria possibilitar a visualização, utilizando o Google Maps e a utilização de diversos filtros on-line;

- Sistema de Visualização de Projetos de Extensão: o sistema deveria ser capaz de importar dados (formatos CSV ou planilhas do Excel) e tratar os mesmos fornecendo uma visualização 
dinâmica dos projetos de extensão desenvolvidos na <universidade>. A visualização deveria fornecer informações para a tomada de decisão (quais são as áreas com maior demanda, locais e público de abrangência, etc);

- Guia Interativo do Estudante: o sistema deveria fornecer um guia interativo para os estudantes da <instituição>, possuindo informações sobre internacionalização e acessibilidade. $\mathrm{O}$ sistema deveria ser em formato de portal e aplicativo para dispositivos móveis (plataforma Android), contando com requisitos de acessibilidade específicos, por exemplo, o uso de tags RFID (Radio FrequencyIdentification), comunicando-se com o celular de uma pessoa com deficiência visual para guiá-la no campus;

- Sistema de Acompanhamento de Estudantes Estrangeiros e de Intercâmbio: atualmente, todo $\mathrm{o}$ acompanhamento dos estudantes que saem da <instituição> para outros lugares e alunos estrangeiros é feito manualmente. A ideia era a de desenvolver um sistema que permitisse o acompanhamento dos alunos e mapas de visualização para a comunidade (onde os alunos estão, de onde vêm os alunos estrangeiros, etc);
- Sistema de Gerenciamento do Trabalho de Conclusão de Curso (TCC): o sistema deveria gerenciar todo o processo do TCC, envolvendo os fluxogramas do acompanhamento do TCC, bem como as avaliações, entregas, etc.

Como cada grupo de alunos possuía um cliente real, foram realizadas 4 entregas no semestre para que os próprios clientes pudessem avaliar o andamento dos projetos e sugerir mudanças e novos requisitos. $\mathrm{Na}$ primeira avaliação ocorreram alguns problemas de comunicação entre grupos e clientes, sendo que um dos clientes apresentou uma expectativa muito diferente do solicitado. Desta forma, um dos grupos foi realocado para outro projeto com um novo cliente. Outro problema enfrentado foram os requisitos que não seriam possíveis de serem implementados, como, por exemplo, a integração do sistema em desenvolvimento com outros sistemas da universidade. Além de complexo, os alunos não tinham acesso e autorização do CPD (Centro de Processamento de Dados) para acessar as bases de dados e sistemas internos.

\section{RESULTADOS OBTIDOS E LIÇÕES APRENDIDAS}

Em todas as edições da disciplina de Projeto de Software houve resultados significativos:

- Todos os sistemas desenvolvidos são 
funcionais, ou seja, os sistemas poderiam (alguns já estão) ser utilizados efetivamente;

- Os projetos demonstram a capacidade dos alunos de Sistemas de informação em desenvolver software para resolver problemas reais;

- Foram publicados dois resumos [ref01, ref03] e dois artigos completos [ref02, ref04] dos projetos desenvolvidos na segunda edição da disciplina;

- A disciplina possibilitou a participação efetiva de todos os alunos. Mesmo os alunos que não gostavam de programar tiveram a oportunidade de trabalhar efetivamente nos projetos, por exemplo, na análise e especificação de requisitos, nos testes, na organização dos artefatos, no gerenciamento do grupo, etc.

Mesmo assim, durante o desenvolvimento dos projetos surgiram vários problemas: desentendimento entre membros do grupo, requisitos especificados de forma errada o que ocasionou retrabalho, problemas na comunicação com os clientes reais, falta de experiência em trabalho em equipe e na utilização correta das técnicas de Engenharia de Software. Tendo como base os principais problemas que surgiram no decorrer da disciplina, temos algumas lições aprendidas:

- Projetos com temas livres atrasaram consideravelmente, pois a demora para a escolha do tema (mesmo com prazos) e incertezas no tema escolhido acabaram atrasando o desenvolvimento;

- Os melhores projetos foram com foco específico (tema e tecnologias) já definido pelo professor como realizado na segunda edição. Cabe ressaltar que a segunda edição da disciplina resultou em 2 artigos completos publicados em anais de um evento científico (ref01, 2015; ref03, 2015);

- Projetos com clientes reais são difíceis, principalmente no entendimento dos requisitos (o que os clientes querem e o que os alunos entendem). Aliado a isso, o prazo de um semestre é um tempo muito pequeno para desenvolver um sistema para um cliente real, que não é da área de informática e cria expectativas, muitas vezes, irreais;

- Realizar reuniões semanais com cada grupo possibilitou o acompanhamento de perto do que cada aluno estava fazendo. Nestas reuniões, cada aluno deveria dizer o que fez na semana anterior, quais as suas atividades a serem realizadas até a próxima semana e se estava enfrentando algum problema;

- Outro aspecto importante foi o de 
utilizar o desenvolvimento de software distribuído com ferramentas utilizadas por empresas, e possibilitar que os alunos, participem de um projeto completo de desenvolvimento de software. A utilização do Githube do Trello motivou os alunos e tornou o desenvolvimento mais dinâmico.

\section{CONCLUSÕES}

Este artigo apresentou as atividades desenvolvidas na disciplina de Projeto de Software em um curso de Sistemas de informação. A disciplina já foi ofertada nos anos de 2013, 2014 e 2015 (uma vez ao ano), com abordagem diferente em cada uma delas. Concluiu-se que a segunda edição da disciplina apresentou os melhores resultados, permitindo a construção de sistemas com todas as funcionalidades previstas implementadas, software com melhor qualidade e artigos publicados.

Acredita-se que os resultados obtidos tenham sido satisfatórios, considerando a avaliação do processo acadêmico realizado pelos alunos, os sistemas desenvolvidos e a produção de artigos científicos sobre os mesmos. Os alunos têm participado de equipes de desenvolvimento de software empregando tecnologias atuais, participando de experiências práticas, que estimulam a integração com o mercado e o processo de gestão de projetos. Estas características (tecnologias atuais, experiências práticas, integração com o mercado, entre outros aspectos) são importantes para a formação do perfil do egresso de SI e devem fazer parte das estruturas curriculares e das experiências dos acadêmicos (SILVEIRA; RIBEIRO, 2009; SILVEIRA, 2009).

Como trabalhos futuros, pretende-se propor para a próxima edição da disciplina, uma abordagem semelhante à segunda edição, com relação ao tema e tecnologias, aliado ao desenvolvimento distribuído de software da terceira edição.

\section{Referências}

ALMENDRA, C. C.; MAGALHÃES, R. P.; ALMEIDA, C. D. A. (2015). Métodos Ágeis em um Núcleo de Práticas Acadêmico: relato de experiência. Anais do WEI 2015.

BARTH, F. J.; BURD, L.; PIMENTEL, M. (2012) Escritório de Projetos: simulando o ambiente de projetos de software em cursos de tecnologia. Anais do WEI 2012.

BORGES, K. S.; CARVALHO, T. P.; MORAES, M. A. C. (2012). Programa de Extensão "Fábrica de Software Acadêmica": contribuindo para a formação profissional na área de informática. Anais do WEI 2012.

MEC Ministério da Educação (2012). Diretrizes Curriculares Nacionais para Cursos de Graduação em Computação. CNE/CES. 2012. Disponível em: <http://portal.mec.gov.br>. Acesso em: fevereiro, 2016.

PADUA, Wilson. Engenharia de Software. Rio de Janeiro: LTC, 3ed, 2009.

PRESSMAN, Roger S. Engenharia de Software. São Paulo: Bookman, 7ed, 2011. 
SILVEIRA, S. R.; RIBEIRO, V. G. Uma

Reflexão sobre a Construção de Currículos de

Cursos de Sistemas de Informação. Anais do

III WEITCHÊ- WORKSHOP DE

EDUCAÇÃO EM INFORMÁTICA DO

RS. Torres-RS: ULBRA, 2009.

SILVEIRA, S. R. A Problemática da

Formação do Docente da Área de Informática.

Educação e Cidadania, n. 11, 2009. Porto

Alegre: UniRitter. Disponível em:

$<$ http://seer.uniritter.edu.br/index.php/educaca oecidadania/article/view/445>. Acesso em

julho de 2015.

SBC Sociedade Brasileira de Computação (2014). WEI Workshop sobre Educação em

Computação. Disponível em:

<http://csbc2014.cic.unb.br/index.php/wei>.

Acesso em fevereiro, 2016.

SOMMERVILLE, Ian. Engenharia de

Software. São Paulo: Person, 8ed, 2007. 